\title{
Screening strategies for adults with type 2 diabetes mellitus: a systematic review protocol
}

Helen Mearns ${ }^{1,2^{*}} \mathbb{D}$, Paul Kuodi Otiku ${ }^{1,2}$, Mary Shelton ${ }^{3}$, Tamara Kredo ${ }^{4,5}$, Benjamin M. Kagina ${ }^{1,2}$ and Bey-Marrié Schmidt ${ }^{4}$

\begin{abstract}
Background: There is limited evidence on whether screening for type 2 diabetes mellitus affects health outcomes. A recent systematic review of randomised clinical trials found only one trial that met their inclusion criteria; therefore, current guidelines for screening interventions for type 2 diabetes mellitus are based on expert opinions and best practice rather than synthesised evidence. This systematic review seeks to collate evidence from nonrandomised studies to investigate the effect of screening for adults with type 2 diabetes on outcomes including diabetes-related morbidity, mortality (all-cause and diabetes-related) and harms.
\end{abstract}

Methods: This systematic review will follow Effective Practice and Organisation of Care (EPOC) guidelines for the synthesis of non-randomised studies. We will search PubMed/MEDLINE, Scopus, Web of Science, CINAHL, Academic Search Premier and Health Source Nursing Academic (from inception onwards). We will include non-randomised trials, controlled before-after studies, interrupted time-series studies, repeated measures studies and concurrently controlled prospective cohort studies. The primary outcome will be diabetes-related morbidity (microvascular complications of diabetic retinopathy, nephropathy or neuropathy or macrovascular complications of non-fatal myocardial infarction, peripheral arterial disease or non-fatal stroke). The secondary outcomes will be mortality (allcause and diabetes-related) and harms of screening strategies to patients (including psychological harms or adverse events following treatments) or to health care system (including resource allocation for false-positives or overdiagnosis). Two reviewers will independently screen all citations and full-text articles. Data will be abstracted by one reviewer and checked by a second. The risk of bias of individual studies will be appraised using the ROBINS-I tool. GRADE will be used to determine the quality of the scientific evidence. If feasible, we will conduct random effects meta-analysis where appropriate. If necessary, analyses will be conducted to explore the potential sources of heterogeneity (e.g. age, sex, socio-economic status, rural versus urban or low-middle income versus high-income country). We will disseminate the findings via publications and through relevant networks.

(Continued on next page)

\footnotetext{
*Correspondence: helen.mearns@gmail.com

${ }^{1}$ Vaccines for Africa Initiative, University of Cape Town, Cape Town, South

Africa

${ }^{2}$ School of Public Health \& Family Medicine, University of Cape Town, Cape

Town, South Africa

Full list of author information is available at the end of the article
}

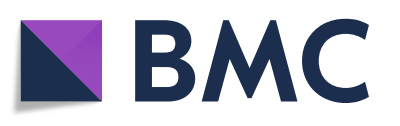

(c) The Author(s). 2020 Open Access This article is licensed under a Creative Commons Attribution 4.0 International License, which permits use, sharing, adaptation, distribution and reproduction in any medium or format, as long as you give appropriate credit to the original author(s) and the source, provide a link to the Creative Commons licence, and indicate if changes were made. The images or other third party material in this article are included in the article's Creative Commons licence, unless indicated otherwise in a credit line to the material. If material is not included in the article's Creative Commons licence and your intended use is not permitted by statutory regulation or exceeds the permitted use, you will need to obtain permission directly from the copyright holder. To view a copy of this licence, visit http://creativecommons.org/licenses/by/4.0/ The Creative Commons Public Domain Dedication waiver (http://creativecommons.org/publicdomain/zero/1.0/) applies to the data made available in this article, unless otherwise stated in a credit line to the data. 
(Continued from previous page)

Discussion: The protocol outlines the methods for systematically reviewing and synthesising evidence of screening strategies for type 2 diabetes mellitus and their effect on health outcomes associated with the disease. The potential impact of this systematic review is improved evidence-informed decision-making for policies and practice for screening of type-2 diabetes.

Systematic review registration: PROSPERO CRD42020147439

Keywords: Screening, Mass screening, Targeted, Opportunistic, Type 2 diabetes mellitus

\section{Background}

\section{Description of the condition}

Diabetes mellitus is a disease of increasing global concern. The global prevalence of diabetes was approximately 425 million people in 2017, approximately $8.5 \%$ of the adult population, and is expected to double by 2045 [1]. In high-income countries, type 2 diabetes mellitus accounts for approximately $90 \%$ of diabetes cases; there is insufficient data to estimate the ratio of type 2 diabetes mellitus in low- and middle- income countries, but it is assumed to be similar [1,2]. Clinical diabetes is diagnosed through the detection of elevated levels of glucose in the blood (hyperglycemia) [3]; however, it is estimated that half of the people who have diabetes are not diagnosed [1].

In addition to those individuals who have clinical diabetes, another 352 million, approximately $7.3 \%$ of the adult population, have intermediate blood glucose levels that are considered in between normal and clinically diagnosed diabetes $[1,3]$. These intermediate blood glucose levels perform as a risk score, where increasing values are associated with an increasing likelihood of progression to diabetes, cardiovascular disease and allcause mortality [2, 4]. Patients who present with intermediate levels of blood glucose are described using a number of terminologies including mild glucose intolerance, non-diabetic hyperglycaemia and prediabetes. The terminology promoted by the World Health Organization (WHO) is impaired glucose tolerance (IGT), impaired fasting glucose (IFG) and intermediate hyperglycaemia $[3,5]$. The term prediabetes is gaining in popularity even though the WHO has warned its use may lead to disease stigma and detract from the significant cardiovascular risk of this population [5]. About a third of people with IGT and IFG are young, aged between 20-39 years, meaning they will spend many years at risk of developing diabetes [1]. Other risk factors, apart from intermediate glucose levels, for the development of diabetes are increasing age of more than 45 years and obesity [2].

Type 2 diabetes mellitus arises due to defective insulin activity in body tissues, defective insulin secretion from pancreas or a combination of the two [2]. Type 2 diabetes mellitus usually occurs in older adults, but with a change in lifestyle factors, such as inactivity and obesity, the condition is increasingly being detected in children, adolescents and young adults [1, 2]. Current management of type 2 diabetes mellitus involves lifestyle modification: increasing physical activity, improving diet, reaching a healthy body weight and stopping smoking, all monitored by regular screening [2]. If lifestyle modification does not result in sufficiently decreased blood glucose levels, medication may be prescribed, of which there are a range of treatment options available [2]. The complication with type 2 diabetes mellitus is the long latency period, often lasting several years, during which time the individual is often asymptomatic and unaware of their condition $[1,2]$. This prolonged asymptomatic state results in long-term damage to the body's organs that leads to negative health outcomes including pregnancy complications, oral health problems, disabilities such as blindness, reduced wound healing, foot disease that may require amputation, stroke, heart and kidney disease and death [1-3].

\section{Description of the intervention}

There are many types of screening interventions and strategies that may be used to detect disease in a population often classified as mass, opportunistic and targeted strategies-as presented in Table $1[2,6]$. This systematic review will use these classifications, but if additional strategies are noted, these too will be included.

The biochemical tests commonly used are fasting plasma glucose (FPG), oral glucose tolerance test (OGTT) and detection of glycated haemoglobin A1C (HbA1c) although there are also urine glucose tests available or random blood glucose tests $[2,6]$. In addition, there are a number of risk scores $[7,8]$, including the Finnish Diabetes Risk Score (FINDRISC) [9] and the American Diabetes Association's risk test [10]; however, these are not commonly used as stand-alone screening tools. Classification of patients post testing can be termed as in the normal range or as having diabetes, impaired glucose tolerance (IGT) or impaired fasting glucose (IFG) (as presented in Table 2) [1, 3]. 
Table 1 Screening strategies applied to detect diabetes

\begin{tabular}{ll}
\hline Mass & Screening of an entire apparently healthy population regardless of risk factors \\
Opportunistic & $\begin{array}{l}\text { Screening of individuals, who may or may not be considered at-risk for diabetes, when presenting for any reason to the health system } \\
\text { or other opportunistic interaction (e.g. HIV testing drive) }\end{array}$ \\
Targeted & Seeking out and screening individuals from a population who are considered at-risk of developing diabetes (e.g. obese, older age) \\
\hline
\end{tabular}

\section{How the intervention might work}

The theory behind screening for type 2 diabetes mellitus is to identify either disease or associated risk factors to initiate preventative measures that can halt, slow or improve the course of disease [11]. Therefore, the earlier the disease is detected, especially where there is high risk of disease, theoretically, the better the expected outcomes. The logic model in Fig. 1 describes a complex system in which the intervention interacts with participants, context, implementation and how these affect the outcomes and the impact of this research [12].

\section{Why it is important to do this review}

Guidelines for screening interventions for type 2 diabetes mellitus, such as those released by the UK National Screening Committee [13], the American Diabetes Association [2] or the Society for Endocrinology, Metabolism and Diabetes of South Africa [14], are based on expert opinion and local practice rather than synthesised evidence. This is because there is limited information to provide evidence about best practice for screening interventions for type 2 diabetes mellitus and even less evidence in low- and middle-income countries [15]. A recently published Cochrane review assessed the effects of any type of screening compared with no screening for type 2 diabetes [16] and found only one trial, the ADDI TION-Cambridge trial [17], that met their inclusion

Table 2 WHO recommended ranges used to classify patients according to blood glucose levels [3]

\begin{tabular}{|c|c|}
\hline \multicolumn{2}{|l|}{ Diabetes } \\
\hline $\begin{array}{l}\text { Fasting plasma } \\
\text { glucose }\end{array}$ & $\geq 7.0 \mathrm{mmol} / \mathrm{L}(126 \mathrm{mg} / \mathrm{dl}) \mathrm{OR}$ \\
\hline $\begin{array}{l}\text { 2-h plasma } \\
\text { glucose* }\end{array}$ & $\geq 11.1 \mathrm{mmol} / \mathrm{L}(200 \mathrm{mg} / \mathrm{dl}) \mathrm{OR}$ \\
\hline $\mathrm{HbA1c}$ & $\geq 6.5 \%$ \\
\hline \multicolumn{2}{|c|}{ Impaired glucose tolerance (IGT) } \\
\hline $\begin{array}{l}\text { Fasting plasma } \\
\text { glucose }\end{array}$ & $<7.0 \mathrm{mmol} / \mathrm{L}(126 \mathrm{mg} / \mathrm{dl})$ AND \\
\hline $\begin{array}{l}\text { 2-h plasma } \\
\text { glucose* }\end{array}$ & $\begin{array}{l}\geq 7.8 \text { and }<11.1 \mathrm{mmol} / \mathrm{L}(140 \mathrm{mg} / \mathrm{dl} \text { and } 200 \mathrm{mg} / \\
\mathrm{dl})\end{array}$ \\
\hline \multicolumn{2}{|c|}{ Impaired fasting glucose (IFG) } \\
\hline $\begin{array}{l}\text { Fasting plasma } \\
\text { glucose }\end{array}$ & $\begin{array}{l}6.1 \text { to } 6.9 \mathrm{mmol} / \mathrm{L}(110 \mathrm{mg} / \mathrm{dl} \text { to } 125 \mathrm{mg} / \mathrm{dl}) \text { AND } \\
\text { (if measured) }\end{array}$ \\
\hline $\begin{array}{l}\text { 2-h plasma } \\
\text { glucose }\end{array}$ & $<7.8 \mathrm{mmol} / \mathrm{L}(140 \mathrm{mg} / \mathrm{dl})$ \\
\hline
\end{tabular}

*Venous plasma glucose $2 \mathrm{~h}$ after ingestion of $75 \mathrm{~g}$ oral glucose load criteria. The ADDITION-Cambridge trial consisted of 20,184 participants aged 40-69 years from general practices in England who were at risk for diabetes but had no known diabetes. These participants were randomised to screening versus no screening arms, and followed up for a median of 9.6 years (November 2001 to November 2011). The review found moderate certainty evidence that screening for diabetes probably makes little or no difference to all-cause mortality and low certainty evidence that it may make little or no difference to diabetes-related mortality. However, because the review only included one trial, firm conclusions about early diabetes screening on health outcomes cannot be drawn. In consultation with the authors of the unpublished Cochrane review and considering the public health importance of screening and the potential impact on large populations, we propose to assess evidence from nonrandomised intervention study designs. The questions for the systematic review will include the following: Does screening for type 2 diabetes mellitus reduce morbidity and/or mortality? Does a particular screening strategy result in a greater reduction of morbidity and/or mortality as compared to another screening strategy? Does screening for type 2 diabetes mellitus result in harms to participants or the health system?

\section{Objectives}

Primary objective

To assess the effectiveness of targeted, opportunistic or mass screening for type 2 diabetes mellitus on reduction of diabetes-associated morbidity in adults

\section{Secondary objectives}

To assess the effectiveness of targeted, opportunistic or mass screening for type 2 diabetes mellitus on reduction of mortality (all cause as well as diabetes-associated) in adults

To assess the harms of targeted, opportunistic or mass screening for type 2 diabetes mellitus in adults

\section{Methods}

The present protocol has been registered within the PROSPERO database (CRD42020147439). This manuscript is being reported in accordance with the reporting guidance provided in the Preferred Reporting Items for Systematic Reviews and Meta-Analyses Protocols (PRIS MA-P) statement [18] (see checklist in Additional file 1). 


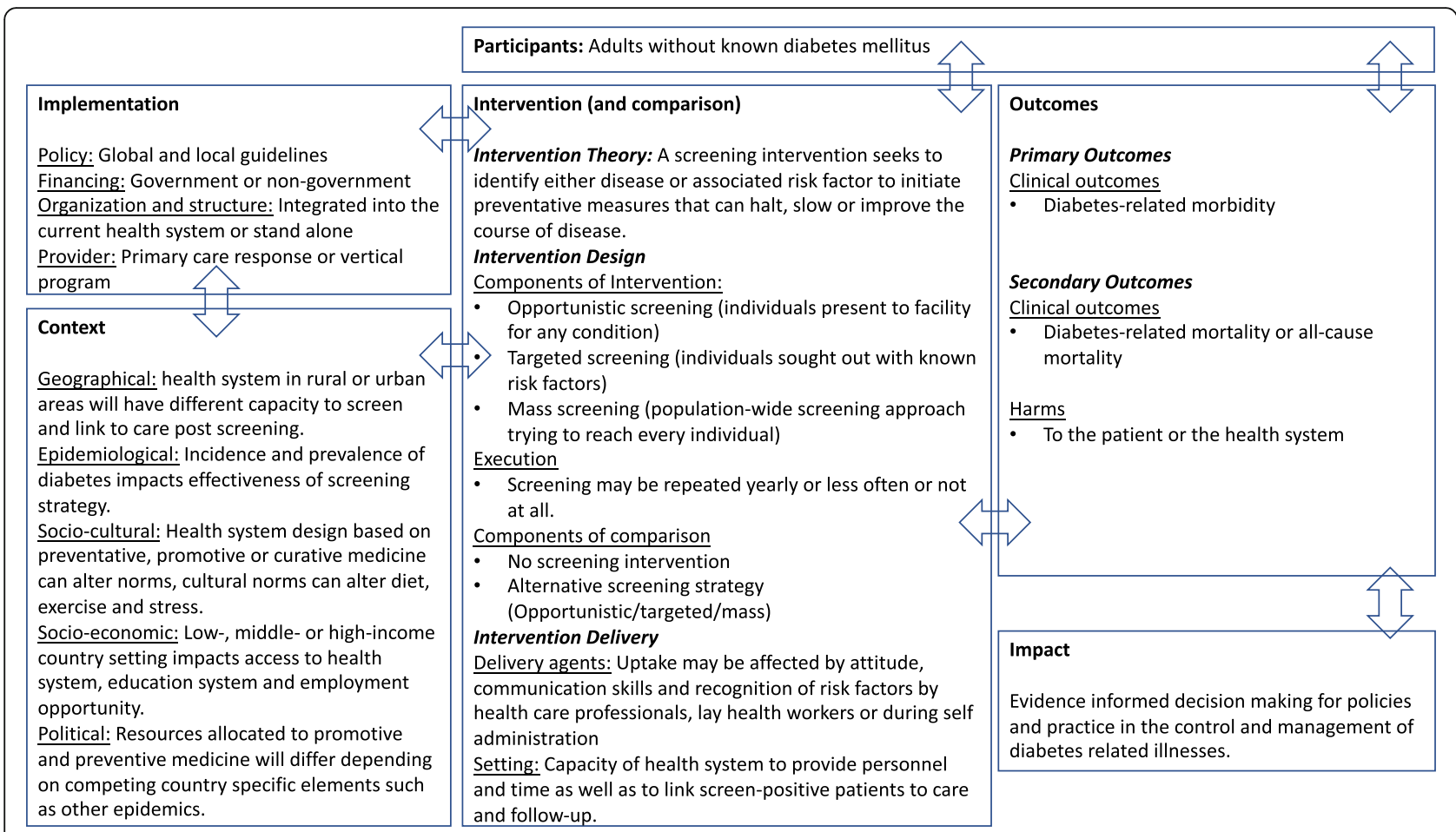

Fig. 1 Logic model describing the interactions between screening for diabetes, implementation, context, participants, outcomes and impact

\section{Study and source eligibility Types of studies}

As existing reviews have found limited randomised evidence addressing this question $[15,19,20]$, we will focus on non-randomised intervention studies (NRIS). We will employ the Cochrane EPOC criteria [21], and NRIS of interest will include non-randomised trials, controlled before-after studies, interrupted time-series study, repeated measures study and concurrently controlled prospective cohort study. The difficulty associated with labelling NRIS is well-documented in the literature; several of these designs, for example, have been used interchangeably; we will make use of the EPOC definitions and flow diagram to assist in study design identification (Appendix 1):

- Non-randomised trial (NRT) is a study design in which individual participants, or clusters of participants, are allocated to intervention or comparator in a quasi-random or non-random manner. If there is an allocation rule, it is often by, for example, alternation, day of the week, odd/even hospital, or identification number.

- Controlled before-after (CBA) is a study design that estimates intervention effectiveness by comparing pre- and post-intervention outcomes in individuals or clusters that receive the intervention and those that do not.
- Interrupted time series (ITS) studies design uses multiple observations from individuals or clusters pre-intervention to establish the preexisting outcome trend; intervention effectiveness is then estimated by measuring postintervention changes in the expected outcome trend associated with the introduction of an intervention (the 'interruption'). An ITS study can identify both immediate and long-term changes associated with the intervention. The interrupted time-series studies will be required to have a clearly defined point in time when the intervention occurred and a minimum of 3 time points before and 3 time points after the intervention [21].

- A repeated measures (RM) study is an interrupted time-series study but where the outcomes of interest are measured in the same participants at each point in time.

- Concurrently controlled prospective cohort study (PCS) is where subjects are identified prospectively as having received an intervention or comparator and are then followed over time. The allocation rule is often in relation to organizational factors such as ward, clinic, doctor or provider organisation. Control arms should be contemporaneous, we will not include retrospective control arms. 
‘PICO' eligibility

Types of participants

We will include adults aged 18 years and older without documented diabetes mellitus or pregnancy.

\section{Types of interventions}

We will include studies comparing one of the screening strategies, targeted, opportunistic or mass screening interventions for the detection of type 2 diabetes mellitus, against no screening or another of the screening strategies (Table 1). There will be a 6-month minimum follow-up time required for the primary clinical outcome of morbidity.

\section{Types of outcome measures}

\section{Primary outcomes Clinical outcomes}

- Diabetes-related morbidity defined as study-reported microvascular complications (diabetic retinopathy, diabetic nephropathy, diabetic neuropathy) or macrovascular complications (non-fatal myocardial infarction, peripheral arterial disease, non-fatal stroke) and measured from 6 months after screening

\section{Secondary outcomes Clinical outcomes}

- Mortality (all-cause and diabetes-related) defined as death due to any-cause including diabetes or other cardiovascular causes (including acute myocardial infarction, ischemic heart disease, stroke or any cardiovascular disorder that lead to death) and measured at any time after screening

\section{Harms of diabetes screening}

Harms to patients is defined as event/s reported in the study at any time after screening.

- Psychological harms such as anxiety or stigma that impacts on quality of life due to a false-positive test

- Number of days of work lost

- Side-effects from treatment

- Loss of health insurance benefits

Harms to health care system is defined as event/s reported in the study at any time after screening.

- False-positive test resulting in human, physical and financial resource allocation to patients who are not in need

- Overdiagnosis may lead to over-extension of human, physical and financial resources for patients who end up in prolonged treatment and engagement with the health system even if they never develop disease
The rationale for prioritisation of outcomes: Primary outcome serves to inform whether screening alters the course of disease as assumed per screening theory [11] and depicted in Fig. 1. Secondary outcome of mortality contributes to the current data outlining no reduction in mortality following screening intervention [19] while also assessing harms that may arise from screening intervention [3] and therefore contribute to evidence to substantiate policy and practice recommendations.

\section{Search methods for identification of studies Electronic searches}

The University of Cape Town Health Sciences Reference Librarian (MS) assisted the first author (HM) in developing the search strategy and will provide advice and guidance in conducting the searches for the review.

\section{Electronic Database Search (from inception onwards) \\ - PubMed (MEDLINE) \\ - Scopus (includes majority of EMBASE contents) \\ - Web of Science Platform (Web of Science Core Collection, Biological Abstracts, SciELO Citation Index) \\ - Academic Search Premier (on the EBSCOhost platform) \\ - CINAHL (on the EBSCOhost platform) \\ - Health Source Nursing Academic (on the EBSCOhost platform)}

A draft search strategy for PubMed/MEDLINE, based on the original search strategy utilised by the Cochrane Review team and revised by an information specialist, is provided in Appendix 2 (see Appendix 2). We will include all studies regardless of publication status; however, we will only include English language studies. We are aware that this decision may lead to language bias [22], but due to capacity and resource limitation of the systematic review team, we are restricted to English only. We will search all databases from inception to the date of search. The search syntax will first be tested and optimised in PubMed. We will thereafter replicate the searches in the other databases adapting search syntax as necessary for those databases.

Grey literature search We will conduct a grey literature search to identify studies not indexed in the databases listed above.

- OpenGrey (multidisciplinary European database, covering science, technology, biomedical science, economics, social science and humanities)

- Conference abstracts from The American Diabetes Association (ADA), the European Association for the Study of Diabetes (EASD) meeting and 
Diabetologia will be used to track down full text articles.

- National Institute for Health Research Economic Evaluation Database (NHS EED)

- Cost-Effectiveness Analysis Registry (CEA) (www.healtheconomics)

We will search key references, such as systematic reviews, by cross-checking reference lists for additional potentially eligible primary studies [23]. We will also contact experts in the field to check if we have missed any relevant studies. We may contact authors of included studies to clarify reported published information and to seek unpublished data.

\section{Methods for screening search results Screening methods}

We will collate and transfer search results to the Rayyan screening software [24] and remove duplicate records. At least two review authors will independently screen titles and abstracts of every record retrieved. Outcome measures will not be used to exclude studies during title and abstract screening. The potentially eligible records will be retrieved for full text screening. The two review authors will independently review full text records for compliance of studies with eligibility criteria of the review. A decision tree based on the eligibility criteria will be used to assist in decision making for exclusion of studies (see Appendix 3). Two review authors will resolve any disagreements through discussion or, if required, will consult a third review author. A study must meet all inclusion criteria to be included. We will list excluded studies at the full text screening stage in the 'Characteristics of excluded studies' table. We will collate multiple reports of the same study so that each study rather than each report is the unit of interest in the review. We will provide any information we can obtain about ongoing studies. We will record the selection process in sufficient detail to complete a PRISMA flow diagram [25].

\section{Data collection and analysis \\ Data extraction}

We will use a standard data extraction form in Microsoft Excel to capture study characteristics and outcome data $[22,26]$; we will pilot the form on at least one eligible study. One review author will extract the following study characteristics from the included studies, and an independent review author will check the extraction:

1. Source: study ID (created by review author), review author ID (created by review author), citation and contact details
2. Eligibility: confirm eligibility for review, reason for exclusion

3. Methods: study design, number of study centres and location, study setting, withdrawals, date of study, follow-up, confounding factors considered, and the methods used to control for confounding, aspects of risk of bias specific for NRIS (see "Assessment of risk of bias in included studies" below), how missing data was handled

4. Participants: number, mean/median age, age range, gender, severity of condition, diagnostic criteria, inclusion criteria, exclusion criteria, screening criteria, diagnostic criteria, presence of known risk factors for type 2 diabetes mellitus (obesity, family history), co-morbidity (hypertension, dyslipidaemia), socio-demographics

5. Interventions: intervention components, comparison, fidelity assessment using the Template for Intervention Description and Replication (TIDieR) as a guide [27]

6. Outcomes: primary and secondary outcomes specified above in the section "Types of outcome measures".

7. Miscellaneous: funding source, notable conflicts of interest of study authors, ethical approval, key conclusions of the study authors, miscellaneous comments from the study authors, references to other relevant studies, correspondence required, miscellaneous comments by the review authors.

One review author will extract outcome data from included studies, and an independent review author will check extracted data. We will note in the 'Characteristics of included studies' table if outcome data were reported in an unusable way. We will resolve disagreements by consensus or by involving a third review author.

\section{Assessment of risk of bias in included studies}

Two review authors will independently assess risk of bias for each study using the ROBINS-I tool [28]. Any disagreement will be resolved by discussion or by involving a third review author.

We will assess the risk of bias according to the following domains:

1. Pre-intervention: bias due to confounding

2. Pre-intervention: bias in selection of participants into the study

3. At intervention: bias in classification of interventions

4. Post-intervention: bias due to deviations from intended interventions

5. Post-intervention: bias due to missing data

6. Post-intervention: bias in measurement of outcomes 
7. Post-intervention: bias in selection of the reported result

We will judge each potential source of bias as low risk, moderate risk, serious risk, critical risk of bias or no information. We will summarise the 'Risk of bias' judgements across different studies for each of the domains listed. We will consider blinding separately for different key outcomes where necessary (e.g. for unblinded outcome assessment, risk of bias for all-cause mortality may be very different than for a patient reported pain scale). Where information on risk of bias relates to unpublished data or correspondence with a trialist, we will note this in the 'Risk of bias' table. We will not exclude studies on the grounds of their risk of bias but will clearly report the risk of bias when presenting the results of the studies. When considering treatment effects, we will take into account the risk of bias for the studies that contribute to that outcome. We will conduct the review according to this published protocol and report any deviations from it in the 'Differences between protocol and review' section of the systematic review.

\section{Dealing with missing data}

Authors will be contacted, and missing data will be requested. If only returned in part and data can be logically imputed, such as standard errors, this will occur. All missing data will be clearly reported in the data extraction forms and risk of bias table and as such be assessed in the sensitivity analysis.

\section{Data management}

EndNote X9 and Microsoft Excel will be used for data management. If there is a conflict between data reported across multiple sources for a single study (e.g. between a published article and a trial registry record), we will report the data from the first peer-reviewed published article.

\section{Data synthesis \\ Preparation for data synthesis}

In preparation for synthesis (either meta-analyses or synthesis without meta-analysis), we will assess how much data are available for each of our objectives by creating a table to compare the PICO elements and the study design features as well as the extracted numerical data for the compilation of a meta-analysis.

\section{Measures of treatment effect}

We will estimate the effect of the intervention using risk ratio for dichotomous data, and mean difference or standardised mean difference for continuous data. Time to event outcomes will be reported as hazard ratios. If other effect estimates are provided, we will convert between estimates where possible. Measures of precision will be $95 \%$ confidence intervals. We will ensure that an increase in scores for continuous outcomes can be interpreted in the same way for each outcome, explain the direction to the reader, and report where the directions were reversed if this was necessary. Interrupted time series data will be analysed and, if required, a statistical comparison of time trends before and after the intervention will be performed. For ITS studies, the guideline as outlined in Analysis in EPOC reviews will be followed with assistance of a statistician to ensure integrity of analysis [29].

\section{Unit of analysis issues}

To avoid unit of analysis errors we will consider the unit used to cluster the intervention (such as a ward, clinic, doctor or provider organisation) or in the case of repeated measures that there will be multiple observations for the same outcome. For instance, multiple screening intervention events per participant may occur over time that may cause a unit-of-analysis error. In order to calculate the confidence intervals, the participants per treatment group rather than the number of intervention attempts will be used [22]. Multiple intervention groups could create unit-of-analysis issues especially if different screening interventions are compared against no screening intervention and use the same participants with no screening intervention in both comparisons [22]. If there is more than one comparison in the study design, we will combine groups into a single pairwise comparison. If there is a unit of analysis error in the reported analysis for a study and there is insufficient information to reanalyse the results, the study authors will be contacted to obtain necessary data. If these data are not available, we will not report confidence intervals or $p$ values for which there is a unit of analysis error [30].

\section{Quantitative synthesis}

We will undertake meta-analyses only where this is meaningful, i.e. if the interventions, participants and the underlying clinical question are similar enough for pooling to make sense. If feasible and appropriate, outcome data from primary studies will be used to perform random effects meta-analyses. Since heterogeneity is expected a priori, we will estimate the pooled treatment effect estimates and its 95\% confidence interval using the random effects model. The random effects model assumes that the effect estimates follow a normal distribution, considering both within-study and between-study variation.

\section{Assessment of heterogeneity}

Forest plots will be used to visualise the extent of heterogeneity among studies. We will quantify statistical 
heterogeneity by estimating the variance between studies using $I^{2}$ statistic. The $I^{2}$ is the proportion of variation in effect estimates that is due to genuine variation rather than sampling (random) error. $I^{2}$ ranges between 0 and $100 \%$ (with values of $0-25 \%$ and $75-100 \%$ taken to indicate low and considerable heterogeneity, respectively) [22]. We will also calculate the chi-squared test where a $p$ value $<0.1$ indicates statistically significant heterogeneity.

\section{Assessment of publication bias}

If we include more than 10 studies investigating a particular outcome, we will use a funnel plot to explore possible publication bias, interpreting the results with caution [31].

\section{Subgroup analysis and investigation of heterogeneity}

We expect the following population characteristics may introduce clinical heterogeneity: age, sex, socioeconomic status [6].

We expect the following contexts may introduce health system heterogeneity: study setting of rural or urban or in a low-middle income country or a highincome country (as defined by the World Bank) [6].

We will use the following outcomes in subgroup analysis:

1. Diabetes-associated morbidity

2. Mortality (all-cause and diabetes-associated)

3. Harms

\section{Sensitivity analysis}

We may conduct a sensitivity analysis to explore the influence of various factors on the effect size of the primary outcomes of the review only. We will stratify studies according to:

1. Restricting the analysis to published studies.

2. Restricting the analysis to studies with a low risk of bias, as specified in "Assessment of risk of bias in included studies"

3. Imputing missing data.

Any post hoc sensitivity analyses that may arise during the review process will be justified in the final report.

\section{Assessment of certainty of evidence using the GRADE approach}

Two review authors will independently assess the certainty of the evidence (high, moderate, low and very low) for each outcome using the five GRADE considerations for downgrading the certainty of evidence (risk of bias, consistency of effect, imprecision, indirectness, and publication bias) and the three criteria for upgrading the certainty of evidence (large effect, dose response and residual confounding opposing the observed effect) [32]. We will use the GRADEpro software GDT [33] to create the 'Summary of findings' tables for the main intervention comparisons and include the following outcomes: diabetes-associated morbidity, mortality (all-cause and diabetes-associated), harms (see Appendix 4 for SoF). We will resolve disagreements on certainty ratings by discussion and provide justification for decisions to down- or upgrade the ratings using footnotes in the SoF table and make comments to aid readers' understanding of the review where necessary. We will use plain language statements to report these findings in the review [34]. The SoF tables will be used to draw conclusions about the certainty of the evidence within the text of the review. If during the review process, we become aware of an important outcome that we failed to list in our planned 'SoF' tables, we will include the relevant outcome and explain the reasons for this is the section 'Differences between protocol and review'.

\section{Discussion}

Systematic reviews of screening for type 2 diabetes have found no evidence that this intervention saves lives [15, $19,20]$; therefore, this review will primarily focus on the impact of screening on the reduction of diabetesassociated morbidities. The impact of this review is synthesised data for the provision of evidence-based decision-making for informing policy and practice around screening strategies for type 2 diabetes mellitus. Important protocol amendments will be documented and noted in the discussion.

\section{Limitations}

The potential limitations of this review at a study (outcome) level include the following: the potential finding of insufficient studies of similar study design and clinical question to synthesise abstracted study data; the overall completeness and applicability of evidence and quality of evidence especially due to the limitation to non-randomised studies due to the lack of randomised studies and therefore the lower quality of evidence; the limitation to English studies only and therefore the potential to miss published research; the limitation of not being able to discern between all-cause mortality and diabetes-related mortality and therefore combining this outcome under one mortality outcome. The potential limitation of this review at a systematic review process level includes the potential biases in the review process such as post hoc analysis and focus of outcome objectives. 


\section{Appendix 1}

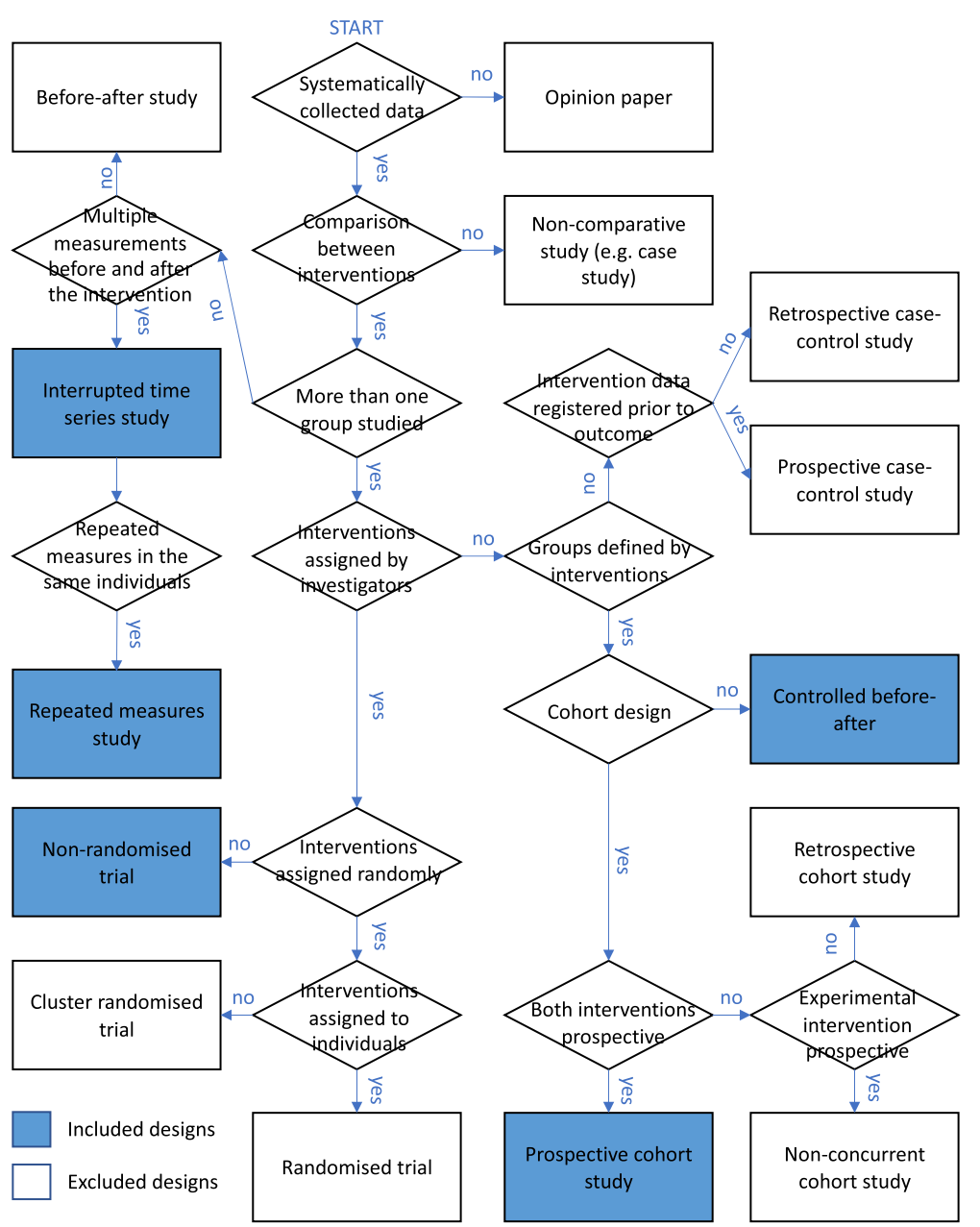

Fig. 2 Flow diagram to assist with identifying the type of study (modified from [34])

\section{Appendix 2}

\section{Search Strategy for PubMed:}

Set 1: Diabetes Mellitus, Type 2 [MeSH] OR [Text Word field:] Adult onset diabetes OR late onset diabetes OR latent diabetes OR mature onset diabetes OR MODY OR NIDDM OR noninsulin-dependent diabetes OR slow onset diabetes OR stable onset diabetes OR type 2 diabetes OR type II diabetes OR T2DM OR T2D

Set 2: Diabetes Insipidus [MeSH] OR [Text Word field:] diabetes insipidus

Set 3: 1 NOT 2

Set 4: Mass screening [MeSH] OR [Text Word field:] screening

Set 5: 3 AND 4

Set 6: Animals [MeSH] NOT Humans [MeSH]

\section{Set 7: 5 NOT 6}

Set 8: [All fields:] Trial OR trials OR before-andafter study OR before-and-after studies OR cohort OR comparative study OR comparative studies OR Controlled OR evaluation study OR evaluation studies OR follow-up study OR follow-up studies OR interrupted time series OR longitudinal study OR longitudinal studies OR non-randomised OR nonrandomized OR nonrandomised OR nonrandomized OR non randomised OR non randomized OR program evaluation OR programme evaluation OR prospective study OR prospective studies OR quantitative study OR quantitative studies OR quasi experimental OR repeated measures

Set 9: 7 AND 8 


\section{Appendix 3}

Table 3 Provisional eligibility decision tree for full text exclusion

\begin{tabular}{|c|c|c|}
\hline Hierarchy & Exclusion reason & Explanation of reason \\
\hline 1 & Duplicate & Record is a duplicate of another study already included in the review \\
\hline 2 & Animal study & Study conducted in non-human population \\
\hline 3 & Study withdrawn & Study was withdrawn before results became available \\
\hline 4 & Ongoing study & $\begin{array}{l}\text { Study is ongoing; No study results have been published. Study will be described in 'Ongoing studies' } \\
\text { section of the review. }\end{array}$ \\
\hline 5 & Wrong intervention & Study does not include screening for type 2 diabetes mellitus \\
\hline 6 & Wrong study design & Study is not eligible as per Appendix 1 study designs. \\
\hline 7 & Wrong population & Study intervention/ outcomes involve individuals who have type 2 diabetes mellitus or are pregnant \\
\hline 8 & $\begin{array}{l}\text { Research question is } \\
\text { inappropriate }\end{array}$ & $\begin{array}{l}\text { Study is not eligible due to inappropriate research question or objectives that do not address systematic } \\
\text { review objectives. }\end{array}$ \\
\hline 9 & Wrong Outcomes & Study does not report outcomes that align with primary or secondary outcome measures. \\
\hline
\end{tabular}

\section{Appendix 4}

Table 4 Provisional summary of findings table

\begin{tabular}{|c|c|c|c|c|c|c|}
\hline \multicolumn{7}{|c|}{ Targeted, opportunistic or mass screening for type 2 diabetes mellitus compared to each other or no screening in children, adolescents and adults. } \\
\hline Patients or population & \multicolumn{6}{|c|}{ Children, adolescents and adults without documented diabetes mellitus or pregnancy. } \\
\hline Intervention & \multicolumn{6}{|c|}{ Targeted, opportunistic or mass screening for type 2 diabetes mellitus. } \\
\hline Comparison & \multicolumn{6}{|c|}{ Other screening (targeted, opportunistic or mass) or no screening for type 2 diabetes mellitus. } \\
\hline \multirow[t]{3}{*}{ Outcomes } & \multicolumn{2}{|c|}{ Illustrative comparative risks (95\% Cl) } & \multirow{3}{*}{$\begin{array}{l}\text { Relative } \\
\text { effect (95\% } \\
\text { Cl) }\end{array}$} & \multirow{3}{*}{$\begin{array}{l}\text { Number of } \\
\text { participants } \\
\text { (studies) }\end{array}$} & \multirow{3}{*}{$\begin{array}{l}\text { Quality of } \\
\text { evidence (GRAD } \\
\text { E) }\end{array}$} & \multirow[t]{3}{*}{ Comments } \\
\hline & Assumed risk & Corresponding risk & & & & \\
\hline & $\begin{array}{l}\text { With targeted, opportunistic } \\
\text { or mass screening }\end{array}$ & $\begin{array}{l}\text { With other screening or } \\
\text { without screening }\end{array}$ & & & & \\
\hline \multicolumn{7}{|l|}{$\begin{array}{l}\text { Diabetes-related } \\
\text { morbidity }\end{array}$} \\
\hline \multicolumn{7}{|l|}{$\begin{array}{l}\text { Mortality (all-cause and } \\
\text { diabetes-related) }\end{array}$} \\
\hline Harms & & & & & & \\
\hline
\end{tabular}

\section{Supplementary information}

Supplementary information accompanies this paper at https://doi.org/10. 1186/s13643-020-01417-3.

\section{Additional file 1. PRISMA Checklist.}

\section{Abbreviations}

ADA: American Diabetes Association; CBA: Controlled before-after; CEA: CostEffectiveness Analysis Registry; CEBHA+: Collaboration for Evidence-Based Healthcare and Public Health in Africa; EASD: European Association for the Study of Diabetes; EPOC: Effective Practice and Organisation of Care; FPG: Fasting plasma glucose; GRADE: Grading of Recommendations Assessment, Development and Evaluation; HbA1c: Detection of glycated haemoglobin A1C; ITS: Interrupted time series; NHS EED: NHS Economic Evaluation Database; NRT: Non-randomised trial; NRIS: Non-randomised intervention studies; OGTT: Oral glucose tolerance test; PCS: Prospective cohort study; PRISMA: Preferred Reporting Items for Systematic Reviews and Meta-Analyses; RM: Repeated measures; SoF: Summary of findings; TIDieR: Template for Intervention Description and Replication

\section{Acknowledgements}

The authors would like to thank the following people who have supported this protocol:

- The authors of an unpublished Cochrane systematic review titled

"Screening for type 2 diabetes mellitus": Nasheeta Peer and Solange Durao,
South African Medical Research Council, for providing their protocol to form the basis of this protocol, as well as a summary of their findings to form the rationale of the protocol and a quote to summarise the findings of their unpublished review.

- Information specialist Maria-Inti Metzendorf, Cochrane Metabolic and Endocrine Disorders Group, provided search strategy for the unpublished

Cochrane review by Peer and Durao upon which the current search strategy is based.

- CEBHA+ Methodological Support Group Jake Burns and Peter Philipsborn, Ludwig-Maximillians Universitat Munchen, are internal reviewers for CEBHA+ which funds this review and assisted by providing critical review of the draft protocol.

- Prof Naomi Levitt, Chronic Disease for Africa, UCT, is a clinical Endocrinologist/Diabetologist who contributed to the PICO of this review when conceptualising the project and gave critical feedback to assist with responding to reviewers comments on the protocol before resubmission.

\section{Authors' contributions}

Conceiving the protocol: BS, TK. Designing the protocol: HM, BS. Coordinating the protocol: HM. Designing search strategies: MS, HM. Writing the protocol: HM. Providing general advice on the protocol and approving the final version: PKO, MS, BK, TK, BS. Securing funding for the protocol: BS, TK. Performing previous work that was the foundation of the current study: NP, SD. Guarantor of the review: BS. The authors read and approved the final manuscript. 


\section{Funding}

The work reported herein was made possible through Cochrane South Africa, South African Medical Research Council under the Collaboration for EvidenceBased Healthcare and Public Health in Africa (CEBHA+) Scholarship Programme. $\mathrm{CEBHA}+$ receives funding from the Federal Ministry for Education and Research (Bundesministerium fur Bildung und Forschung, BMBF), Germany, through the BMBF funding of Research Networks for Health Innovation in Sub-Saharan Africa. The content hereof is the sole responsibility of the authors and does not necessarily represent the official views of SAMRC or the funders. The funding body, the Federal Ministry for Education and Research (Bundesministerium fur Bildung und Forschung, BMBF), Germany, played no role in the design and writing of the protocol; however, members of the Collaboration for Evidence-Based Healthcare and Public Health in Africa (CEBHA+) provided critical review and expert advice on the penultimate version of the protocol.

\section{Availability of data and materials}

Not applicable.

\section{Ethics approval and consent to participate}

Ethics approval is not required for a systematic review of secondary data.

\section{Consent for publication}

Not applicable.

\section{Competing interests}

The authors declare that they have no competing interests.

HM: None known

PKO: None known

MS: None known

BMK: None known

TK: None known

BS: None known

\section{Author details}

${ }^{1}$ Vaccines for Africa Initiative, University of Cape Town, Cape Town, South Africa. ${ }^{2}$ School of Public Health \& Family Medicine, University of Cape Town, Cape Town, South Africa. ${ }^{3}$ Health Sciences Library, University of Cape Town, Cape Town, South Africa. ${ }^{4}$ Cochrane South Africa, South African Medical Research Council, Cape Town, South Africa. ${ }^{5}$ Division of Clinical Pharmacology, Department of Medicine, Faculty of Medicine and Health Sciences, University of Stellenbosch, Cape Town, South Africa.

Received: 21 December 2019 Accepted: 30 June 2020

Published online: 13 July 2020

\section{References}

1. Ogurtsova K, da Rocha Fernandes JD, Huang Y, Linnenkamp U, Guariguata $L$, Cho NH, et al. IDF Diabetes Atlas: global estimates for the prevalence of diabetes for 2015 and 2040. Diabetes Res Clin Pract. 2017;128:40-50.

2. American Diabetes Association. 2. Classification and diagnosis of diabetes: standards of medical care in diabetes-2018. Diabetes Care. 2018;41(Suppl 1):S13-27.

3. World Health Organization. Global report on diabetes. 2016.

4. Sorkin JD, Muller DC, Fleg $J$, Andres R. The relation of fasting and 2-h postchallenge plasma glucose concentrations to mortality: data from the Baltimore Longitudinal Study of Aging with a critical review of the literature. Diabetes Care. 2005;28(11):2626-32.

5. World Health Organization, International Diabetes Federation. Definition and diagnosis of diabetes mellitus and intermediate hyperglycaemia : report of a WHO/IDF consultation. World Health Organization. 2006.

6. World Health Organization. Screening for type 2 diabetes. Geneva, Switzerland: Department of Noncommunicable Disease Management; 2003.

7. Schulze MB, Hoffmann K, Boeing H, Linseisen J, Rohrmann S, Mohlig M, et al. An accurate risk score based on anthropometric, dietary, and lifestyle factors to predict the development of type 2 diabetes. Diabetes Care. 2007;30(3):510-5.

8. Muhlenbruch K, Ludwig T, Jeppesen C, Joost HG, Rathmann W, Meisinger C, et al. Update of the German Diabetes Risk Score and external validation in the German MONICA/KORA study. Diabetes Res Clin Pract. 2014;104(3):459-66.

9. Jolle A, Midthjell K, Holmen J, Carlsen SM, Tuomilehto J, Bjorngaard JH, et al. Validity of the FINDRISC as a prediction tool for diabetes in a contemporary Norwegian population: a 10-year follow-up of the HUNT study. BMJ Open Diabetes Res Care. 2019;7(1):e000769.
10. American Diabetes Association. Screening for diabetes. Diabetes Care. 2002; 25(suppl 1):s21-s4

11. Speechley M, Kunnilathu A, Aluckal E, Balakrishna MS, Mathew B, George EK. Screening in public health and clinical care: similarities and differences in definitions, types, and aims - a systematic review. J Clin Diagn Res. 2017; 11(3):LE01-LE4.

12. Rohwer A, Pfadenhauer L, Burns J, Brereton L, Gerhardus A, Booth A, et al. Series: clinical epidemiology in South Africa. Paper 3: logic models help make sense of complexity in systematic reviews and health technology assessments. J Clin Epidemiol. 2017;83:37-47.

13. Waugh NR, Shyangdan D, Taylor-Phillips S, Suri G, Hall B. Screening for type 2 diabetes: a short report for the National Screening Committee. Health Technol Assess. 2013:17(35):1-90.

14. Amod A. The 2017 SEMDSA guideline for the management of type 2 diabetes. JEMDSA. 2017:22(1):S1-S196.

15. Selph S, Dana T, Blazina I, Bougatsos C, Patel H, Chou R. Screening for type 2 diabetes mellitus: a systematic review for the U.S. Preventive Services Task Force. Ann Intern Med. 2015;162(11):765-76.

16. Peer N, Balakrishna Y, Durao S. Screening for type 2 diabetes mellitus. Cochrane Database Syst Rev. 2020;5:CD005266.

17. Simmons RK, Echouffo-Tcheugui JB, Sharp SJ, Sargeant LA, Williams KM, Prevost AT, et al. Screening for type 2 diabetes and population mortality over 10 years (ADDITION-Cambridge): a cluster-randomised controlled trial. Lancet. 2012;380(9855):1741-8.

18. Moher D, Shamseer L, Clarke M, Ghersi D, Liberati A, Petticrew M, et al. Preferred reporting items for systematic review and meta-analysis protocols (PRISMA-P) 2015 statement. Syst Rev. 2015;4:1.

19. Saquib N, Saquib J, loannidis JP. Does screening for disease save lives in asymptomatic adults? Systematic review of meta-analyses and randomized trials. Int J Epidemiol. 2015;44(1):264-77.

20. Durao S, Ajumobi O, Kredo T, Naude C, Levitt NS, Steyn K, et al. Evidence insufficient to confirm the value of population screening for diabetes and hypertension in low- and-middle-income settings. S Afr Med J. 2015;105(2):98-102.

21. Cochrane Effective Practice and Organisation of Care (EPOC). EPOC review and what should they be called? EPOC resources for review authors. 2017.

22. Higgins JPT, Thomas J, Chandler J, Cumpston M, Li T, Page MJ, et al. Cochrane handbook for systematic reviews of interventions 2019 July 2019.

23. Horsley T, Dingwall O, Sampson M. Checking reference lists to find additional studies for systematic reviews. Cochrane Database Syst Rev. 2011:8:MR000026.

24. Ouzzani M, Hammady H, Fedorowicz Z, Elmagarmid A. Rayyan-a web and mobile app for systematic reviews. Syst Rev. 2016;5(1):210.

25. Liberati A, Altman DG, Tetzlaff J, Mulrow C, Gotzsche PC, loannidis JP, et al. The PRISMA statement for reporting systematic reviews and meta-analyses of studies that evaluate health care interventions: explanation and elaboration. PLoS Med. 2009;6(7):e1000100.

26. Cochrane Effective Practice and Organisation of Care (EPOC). Data collection form. 2013

27. Hoffmann TC, Glasziou PP, Boutron I, Milne R, Perera R, Moher D, et al. Better reporting of interventions: template for intervention description and replication (TIDieR) checklist and guide. BMJ : British Medical Journal. 2014;348:g1687.

28. Sterne JA, Hernan MA, Reeves BC, Savovic J, Berkman ND, Viswanathan M, et al. ROBINS-l: a tool for assessing risk of bias in non-randomised studies of interventions. BMJ. 2016:355:i4919.

29. Cochrane Effective Practice and Organisation of Care (EPOC). Interrupted time series (ITS) analyses. 2017.

30. Cochrane Effective Practice and Organisation of Care (EPOC). Analysis in EPOC reviews. 2017.

31. Sterne JA, Sutton AJ, loannidis JP, Terrin N, Jones DR, Lau J, et al. Recommendations for examining and interpreting funnel plot asymmetry in meta-analyses of randomised controlled trials. BMJ. 2011;343:d4002.

32. Guyatt GH, Oxman AD, Vist GE, Kunz R, Falck-Ytter Y, Alonso-Coello P, et al. GRADE: an emerging consensus on rating quality of evidence and strength of recommendations. BMJ. 2008;336(7650):924-6.

33. McMaster University. GRADEpro GDT. In: Group HOGW, editor. 2014

34. Cochrane Effective Practice and Organisation of Care (EPOC). EPOC worksheets for preparing a 'Summary of findings' table using GRADE. 2013.

\section{Publisher's Note}

Springer Nature remains neutral with regard to jurisdictional claims in published maps and institutional affiliations. 